\title{
REVIEW
}

\section{Visualizing Non-Gaussian Diffusion: Clinical Application of q-Space Imaging and Diffusional Kurtosis Imaging of the Brain and Spine}

\author{
Masaaki Hori $^{1 *}$, Issei Fununaga ${ }^{1,2}$, Yoshitaka MASUTANI ${ }^{3}$, Toshiaki TAOKA ${ }^{4}$, \\ Koji Kamagata $^{1}$, Yuriko Suzuki ${ }^{5}$, and Shigeki Aoki ${ }^{1}$ \\ ${ }^{1}$ Department of Radiology, School of Medicine, Juntendo University \\ 2-1-1 Hongo, Bunkyo-ku, Tokyo 113-8421, Japan \\ ${ }^{2}$ Department of Health Science, Graduate School of Human Health Sciences, Tokyo Metropolitan University \\ ${ }^{3}$ Division of Radiology and Biomedical Engineering, Graduate School of Medicine, The University of Tokyo \\ ${ }^{4}$ Department of Radiology, Nara Medical University \\ ${ }^{5}$ Philips Electronics Japan, Ltd.
}

(Received June 5, 2012; Accepted July 11, 2012)

\begin{abstract}
Recently, non-Gaussian diffusion-weighted imaging (DWI) techniques, including qspace imaging (QSI) and diffusional kurtosis imaging (DKI), have emerged as advanced methods to evaluate tissue microstructure in vivo using water diffusion. QSI and DKI have shown promising results in clinical applications, such as in the evaluation of brain tumors (e.g., grading gliomas), degenerative diseases (e.g., specific diagnosis of Parkinson disease), demyelinating diseases (e.g., assessment of normal-appearing tissue of multiple sclerosis), and cerebrovascular diseases (e.g., assessment of the microstructural environment of fresh infarctions). Representative metrics in clinical use are the full width at half maximum, also known as the mean displacement of the probability density function curve, which is derived from QSI, and diffusional kurtosis, which is derived from DKI. These new metrics may provide information on tissue structure in addition to that provided by conventional Gaussian DWI investigations that use the apparent diffusion coefficient and fractional anisotropy, recognized indices for evaluating disease and normal development in the brain and spine. In some clinical situations, sensitivity for detecting pathological conditions is higher using QSI and DKI than conventional DWI and diffusion tensor imaging (DTI) because DWI and DTI calculations are based on the assumption that water molecules follow a Gaussian distribution, whereas hindrance of the distribution of water molecules by complex and restricted structures in actual neural tissues produces distributions that are far from Gaussian. We review the technical aspects and clinical applications of QSI and DKI, focusing on clinical use and in vivo studies and highlighting differences from conventional diffusional metrics.
\end{abstract}

Keywords: diffusion-weighted imaging, kurtosis, MRI, non-Gaussian, q-space imaging

\section{Introduction}

Diffusion-weighted imaging (DWI) is widely applied as a noninvasive magnetic resonance (MR) technique for evaluating neural tissue in vivo. A DWI-derived parameter, the apparent diffusion coefficient (ADC), has been widely used for assessment.

Water diffusion in tissue is affected by the pres-

\footnotetext{
*Corresponding author, Phone: + 81-3-3813-3111, Fax: + 81-
} 3-3816-0958, E-mail: mahori@juntendo.ac.jp ence and orientation of barriers to translational motion, such as cell membranes and myelin fibers, and is thus sensitive to pathophysiological processes, such as cerebral ischemia, that modify the integrity of these structures. ${ }^{1}$ The measured ADC value of water can vary depending upon the orientation of restricting barriers, such as white matter tracts, relative to the direction of the motion-probing gradient (MPG). After measuring the full diffusion tensor, ${ }^{2}$ we can calculate the trace $[\operatorname{Tr}(\mathrm{ADC})]$ or the average diffusivity $\left[\mathrm{D}=\left(\lambda_{1}+\lambda_{2}+\lambda_{3}\right) / 3\right.$, where $\lambda_{1}, \lambda_{2}$, and $\lambda_{3}$ are the eigenvalues of the diffusion 
tensor], which removes the effects of anisotropy and myelin fiber orientation from the ADC measurement. Using the full tensor data, fractional anisotropy (FA) can be calculated for the index of the white matter anisotropic properties.

These 2 metrics, ADC and FA, are now widely used to diagnose or evaluate disorders of the central nervous system, including stroke, ${ }^{3}$ multiple sclerosis, ${ }^{4}$ neoplasms, ${ }^{5-7}$ neuropsychiatric disease, ${ }^{8-10}$ and degenerative disease $\mathrm{e}^{11-14}$ in clinical situations. Despite their usefulness, however, FA and ADC have limitations. One limitation is that FA and ADC, as conventional diffusion metrics, are calculated based on the assumption of a Gaussian shape for the underlying probability density function (PDF) of the diffusion of water molecules. However, neuronal tissue is a complex environment, and many factors affect the decay of the diffusion signal, such as water restriction by neural structures and intra- and extracellular water exchange ${ }^{15}$ as well as variation in tissue compartment sizes. Therefore, more dedicated, model-free (non-Gaussian) methods for diffusion have been introduced, and reports of their clinical use have recently increased in number. The most popular methods are measurements of mean displacement (MDP, also known as root mean square displacement) of water molecules using q-space imaging (QSI) and diffusional kurtosis (DK) analysis using diffusional kurtosis imaging (DKI). In this article, we briefly explain the principles of QSI and DKI and address the limitations of ADC and FA in various clinical situations and the advantages of MDP and DK over $\mathrm{ADC}$ and FA.

\section{Q-space Analysis}

A non-Gaussian diffusion analysis using multiple b-values, including high b-values, has been introduced to estimate real structural information ${ }^{16-23}$; this is called "q-space imaging." The main principle in q-space analysis is that a Fourier transformation of the signal intensity with respect to $q$, $\mathrm{Et}_{\text {dif }}(\mathrm{q})$, provides the PDF, Ps $\left(\mathrm{R}, \mathrm{t}_{\text {dif }}\right)$, for the water diffusion: $\operatorname{Ps}\left(\mathrm{R}, \mathrm{t}_{\text {dif }}\right)=\mathrm{FT}\left\{\mathrm{E}_{\mathrm{tdif}}(\mathrm{q})\right\},{ }^{21}$ where $\mathrm{R}=$ the distance a spin diffuses during the allowed diffusion time; $\mathrm{t}_{\mathrm{dif}}=\Delta-\mathrm{d} / 3$, the effective diffusion time; $\Delta=$ the time between MPG pulses; and $d=$ the length of the MPG pulse.

Moreover, the shape of the PDF curvature is characterized by the full width at half maximum (FWHM; Fig. 1). The MDP or root mean square displacement value at each MPG axis can be calculated using the following equation ${ }^{21,23}$ : MDP value $=0.425 \times$ FWHM. The normal MDP in the white matter and grey matter is reported to be $3.3 \pm 0.8$ and $7-9,{ }^{19} 3.8 \pm 0.2$ and $5-9,246.6 \pm 0.2$ and $8.44 \pm$ $0.41,{ }^{22} 9$ and $11.9 \mu \mathrm{m} .^{25}$

This method is theoretically superior to conventional Gaussian diffusion analysis, but few reports apply it to human studies ${ }^{21,26}$ because QSI is timeconsuming for daily clinical use, generally taking a minimum of $10 \mathrm{~min}$.

\section{Diffusional Kurtosis Analysis}

DKI has a close relationship to q-space imaging, and QSI methods have indeed recently been employed to estimate diffusional kurtosis. Kurtosis is a dimensionless statistical metric for quantifying the non-Gaussianity of an arbitrary probability distribution. If $\mathrm{Mn}$ is the nth moment of a distribution about its mean value, then the kurtosis may be defined as $\mathrm{K}=\mathrm{M}_{4} / \mathrm{M}_{2}^{2}-3 .{ }^{27}$ The metrics of kurtosis described by Jensen and associates ${ }^{28}$ do not need the full diffusion displacement probability distribution, so the technique is less demanding than QSI in terms of imaging time and gradient strengths.

The important point of Jensen's kurtosis is that the excess DK may be approximately determined from just the first 3 terms of an expansion of the logarithm of the nuclear MR signal intensity in powers of $b$. This is why DK measurement requires only modest increases in b-values beyond those typically employed for DWI. ${ }^{28}$ Parametric maps of Dapp and Kapp were created by fitting the image signal intensities on a voxel-by-voxel basis to the following formula ${ }^{28}: S_{\exp }=\left\{\eta^{2}+\left[S_{0} \exp (-b D\right.\right.$ app $+1 / 6 \times \mathrm{b}^{2} D^{2}$ appKapp) $\left.]^{2}\right\}^{1 / 2}$, where $S_{\text {exp }}$ is the experimental signal intensity, $\eta$ is the background noise, Dapp is an estimate for the diffusion coefficient in the direction parallel to the orientation of diffusion sensitizing gradients, and Kapp is an estimate for the diffusional kurtosis in this same direction. Moreover, additional DKI metrics of mean kurtosis (MK, also known as mean DK), axial kurtosis $\left(\mathrm{K}_{\|}\right)$, and radial kurtosis $\left(\mathrm{K}_{\perp}\right)$ are determined as follows. MK is defined as the average of the kurtosis over all possible diffusion directions; $K_{\|}$is defined, by analogy to $D_{\|}$, as the kurtosis in the direction of the diffusion tensor eigenvector with the largest diffusion eigenvalue; and $K_{\perp}$ is defined as the average of the kurtosis over all directions perpendicular to the diffusion eigenvector with the largest eigenvalues. ${ }^{29}$ Figure 2 shows maps of these metrics. Jensen and Helpern emphasized that, unlike in QSI, the maximum b-value for DKI should be carefully chosen because the DKI formula is valid only for DWI data obtained in normal brain tissue with $\mathrm{b}<3500 \mathrm{~s} / \mathrm{mm}^{227}$ (Fig. 3). In ad- 
DWI data with multiple b values

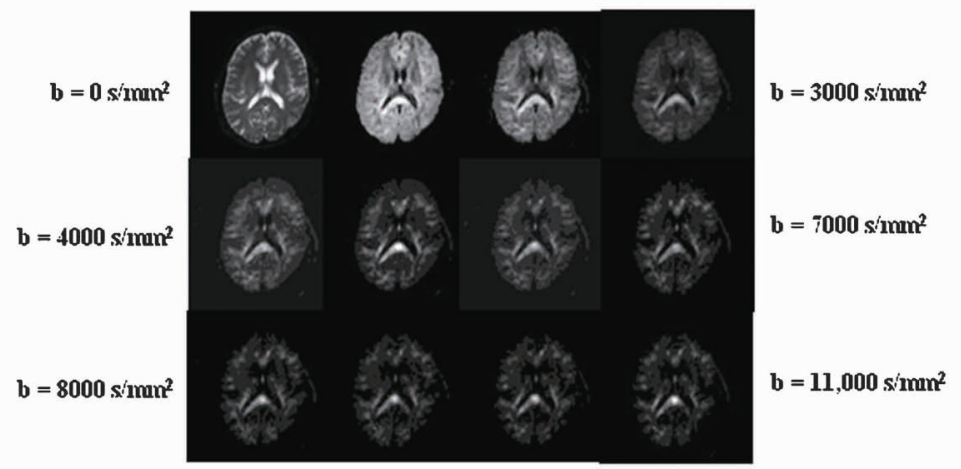

\section{Signal-intensity curves}
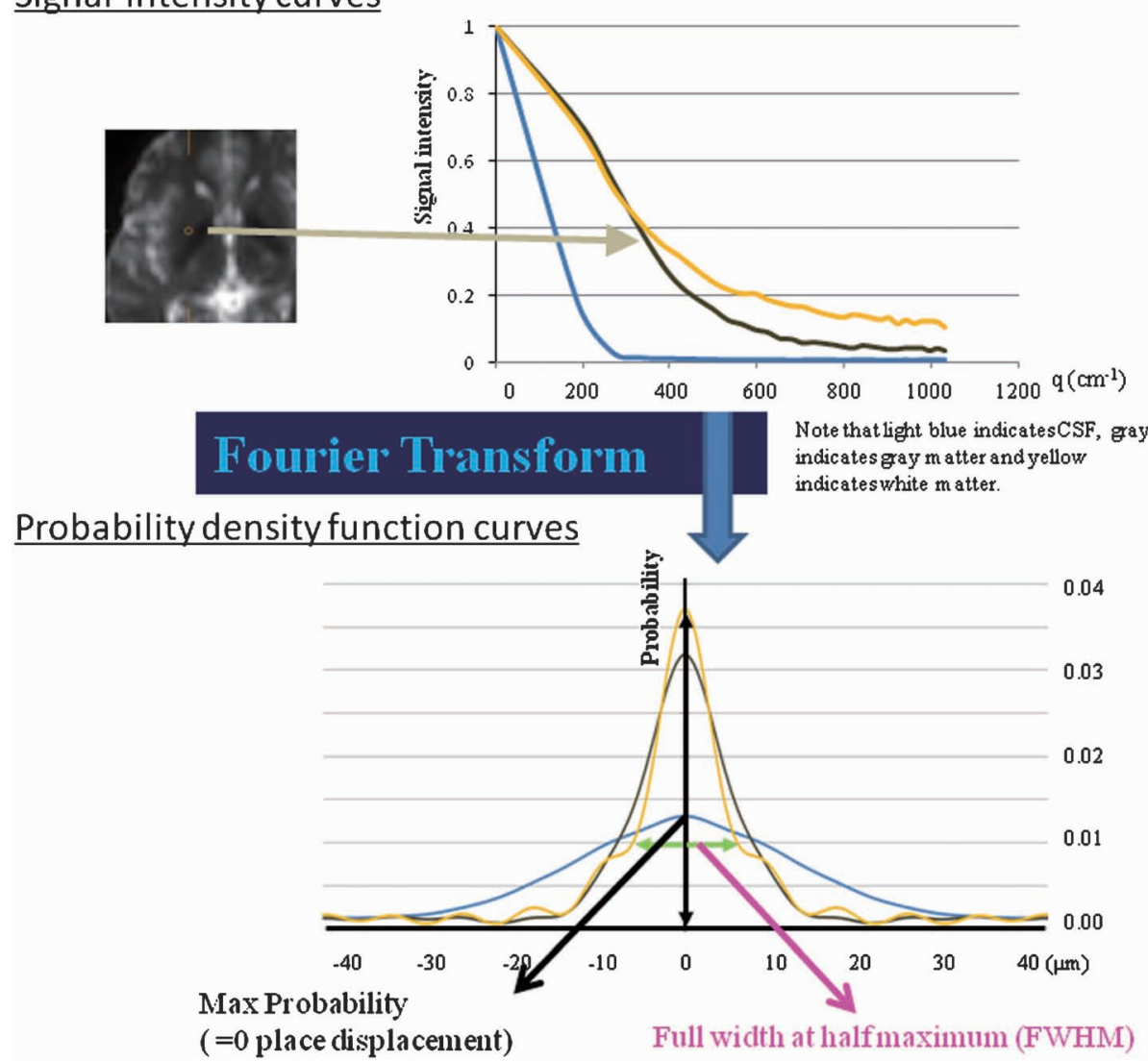

Fig. 1. The process of calculating q-space imaging (QSI) metrics from a diffusion-weighted imaging (DWI) dataset. First, a DWI dataset with multiple b- (q-) values was acquired. From left to right, top to bottom, the images reflect b-values of $0,1000,2000,3000, \cdots 11,000 \mathrm{~s} / \mathrm{mm}^{2}$. A q-value signal intensity curve was obtained at each pixel. After the Fourier transformation of the signal decay with respect to q produced a non-Gaussian displacement distribution profile for each pixel in the image, probability density function curves were calculated. The mean displacement (calculated from the full width at half height) and the probability for zero displacement (given by the height of the profile at zero displacement) are important indices. The yellow line indicates white matter (WM), the gray line indicates gray matter (GM), and the blue line indicates cerebrospinal fluid (CSF).

dition, combinations and maximum b-values used for the DKI should be customized when studying pathological conditions because use of different combinations of b-values will yield different kurto- sis values.

\section{Software for Calculating Non-Gaussian Metrics}

There is no standard software for calculating 


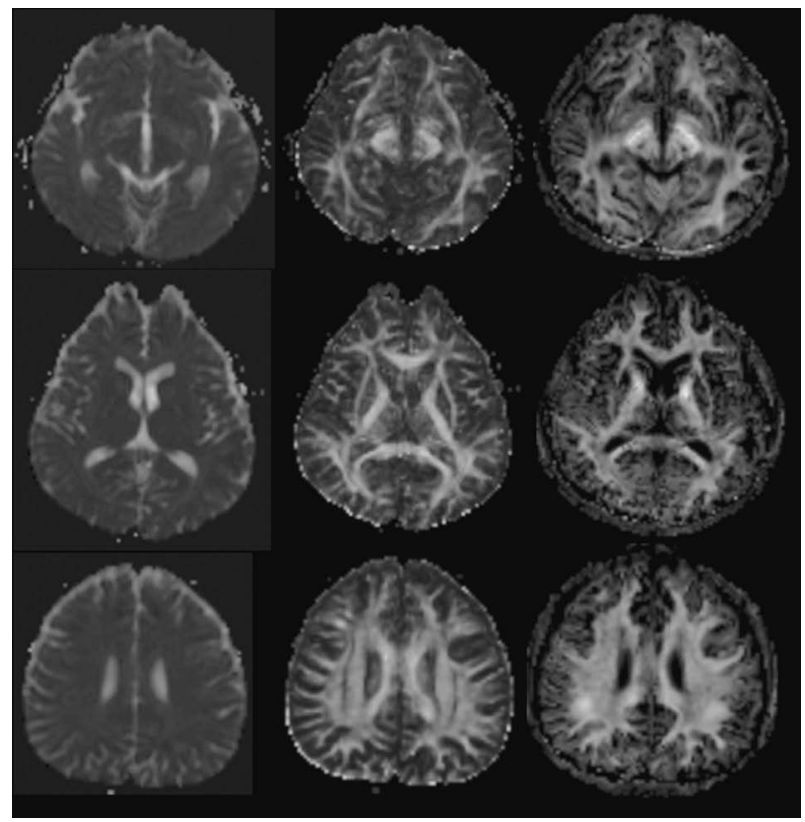

Fig. 2. Left to right columns, apparent diffusion coefficient (ADC), fractional anisotropy (FA), and mean diffusional kurtosis (DK) maps of the brain of a healthy volunteer. Note the similar contrast of the brain structure in the mean DK map and the FA map, but the maps reflect different statuses of brain microstructures because each is derived from a different formula.

non-Gaussian metrics; published reports have used original, in-house software or routines. ${ }^{18,21}$ However, for q-space analysis, QSI-analyzer was introduced for calculating the FWHM of the PDF and the mean displacement of water molecules..$^{22,26,30,31}$ For diffusional kurtosis analysis, diffusion kurtosis estimator (DKE) ) $^{29,32}$ and the combination of dTV $\|$ FZR (http://www.ut-radiology. umin.jp/people/masutani/dTV.htm) and VOLUMEONE software (http://www.volume-one.org/) have been introduced in the literature ${ }^{33,34}$ (Fig. 4).

\section{Clinical Application}

\section{Cerebral ischemia}

ADC values in acute stroke may show an early decrease due to cell depolarization and cytotoxic edema over minutes to one hour, when $\mathrm{T}_{2}$-weighted imaging may still be normal; "pseudonormalization" after one to 10 days due to increased extracellular water content (vasogenic edema); and then a further rise above normal ranges due to cell lysis and necrosis. ${ }^{35,36}$ It is an oversimplification to associate ADC decreases with cerebral ischemia in general; a decrease in ADC is an indicator of ischemia, but it is neither specific to irreversible tissue damage nor does it occur under all circumstances. ${ }^{37}$ In human stroke, the degree to which ADC decreases is correlated with ischemic impairment but does not predict the fate of the tissue after potential reperfusion. ${ }^{37}$

Changes of FA value have often been used to evaluate white matter damage resulting from specific lesions, particularly of major bundles. In addition to the FA values themselves, 3-dimensional tractography calculated using the FA values has also been used to evaluate small lacunar infarctions near the corticospinal tracts; the relationship between the tract and fresh infarction correlates well with motor function 2 weeks later. ${ }^{38}$ Three-dimensional diffusion tensor tractography allowed us to measure the FA values of the corticospinal tracts selectively with an optimal tractability threshold (FA values of about 0.20 ). ${ }^{39}$ However, there have been few studies of ischemic lesions themselves using FA values. Morita and colleagues reported that significant changes in diffusion anisotropy were limited to the severely infarcted core of the white matter, ${ }^{40}$ and those changes were observed in only 3 patients. Assaf and Pasternak reported apparently limited radiological benefits of diffusion tensor imaging (DTI). ${ }^{41}$ In the vast majority of diseases, reduced FA also characterizes abnormal white matter, typically defined as such from $\mathrm{T}_{2}$-weighted MR images. In areas of normal-appearing white matter (NAWM), FA measurement shows significant changes not apparent on conventional MR imaging, but these are typically small and may be less apparent on a single-subject level.

In an experiment using stroke-prone spontaneously hypertensive rats (SPSHR), which develop multifocal stroke lesions in the brain subsequent to hypertension, Assaf and associates found that metrics obtained using a low b-value dataset produced fewer significant changes than those obtained using a high $b$-value q-space diffusion image. ${ }^{42}$ The qspace MDP was significantly higher in the white matter of the SPSHR group than the control group. Electron microscopy also showed significant demyelination in the spinal cords of the SPSHR. The results may indicate that high b-value QSI might be a useful method for observing demyelination and axonal loss. Hori and colleagues ${ }^{26}$ compared MDP values using q-space analysis with ADC using conventional Gaussian metrics in 22 acute or subacute ischemic lesions and found no correlation between ADC and MDP values; though ADC values decreased in all lesions, most lesions $(16 / 22)$ had higher MDP values than normal brain tissue. A more detailed study is needed to evaluate these maps as potential biomarkers for patients 


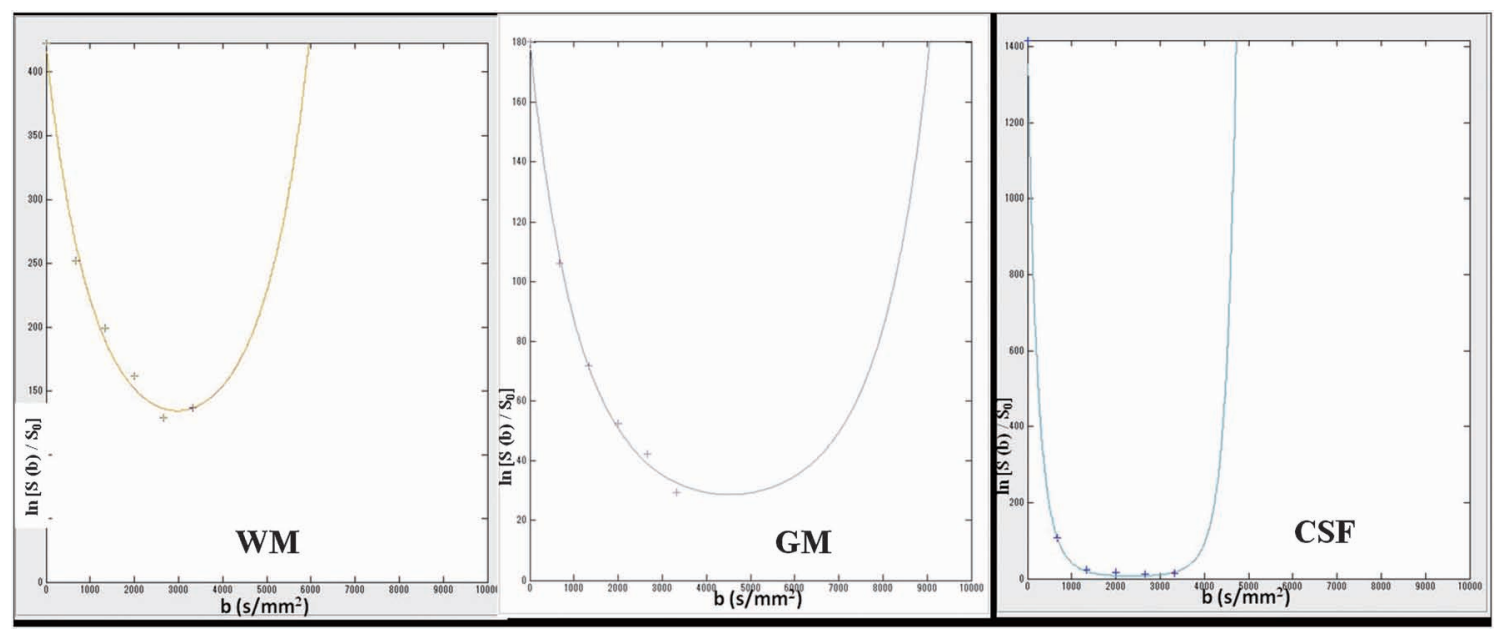

Fig. 3. Diffusional kurtosis imaging (DKI) curve fitting using diffusion-weighted imaging (DWI) data with b-values of $0,667,1333,2000,2667$, and $3333 \mathrm{~s} / \mathrm{mm}^{2}$ in white matter (WM), gray matter (GM), and cerebral spinal fluid (CSF). The upper bound of the curve was seen at $b>2972 \mathrm{~s} / \mathrm{mm}^{2}$ in WM, $4528 \mathrm{~s} / \mathrm{mm}^{2}$ in GM, and $2360 \mathrm{~s} / \mathrm{mm}^{2}$ in CSF. Therefore, for example, DWI data with a bvalue of $3333 \mathrm{~s} / \mathrm{mm}^{2}$ should be excluded from the calculation for accurate parameter estimation in WM. An in-house Matlab program is then used to calculate the fitting curve.

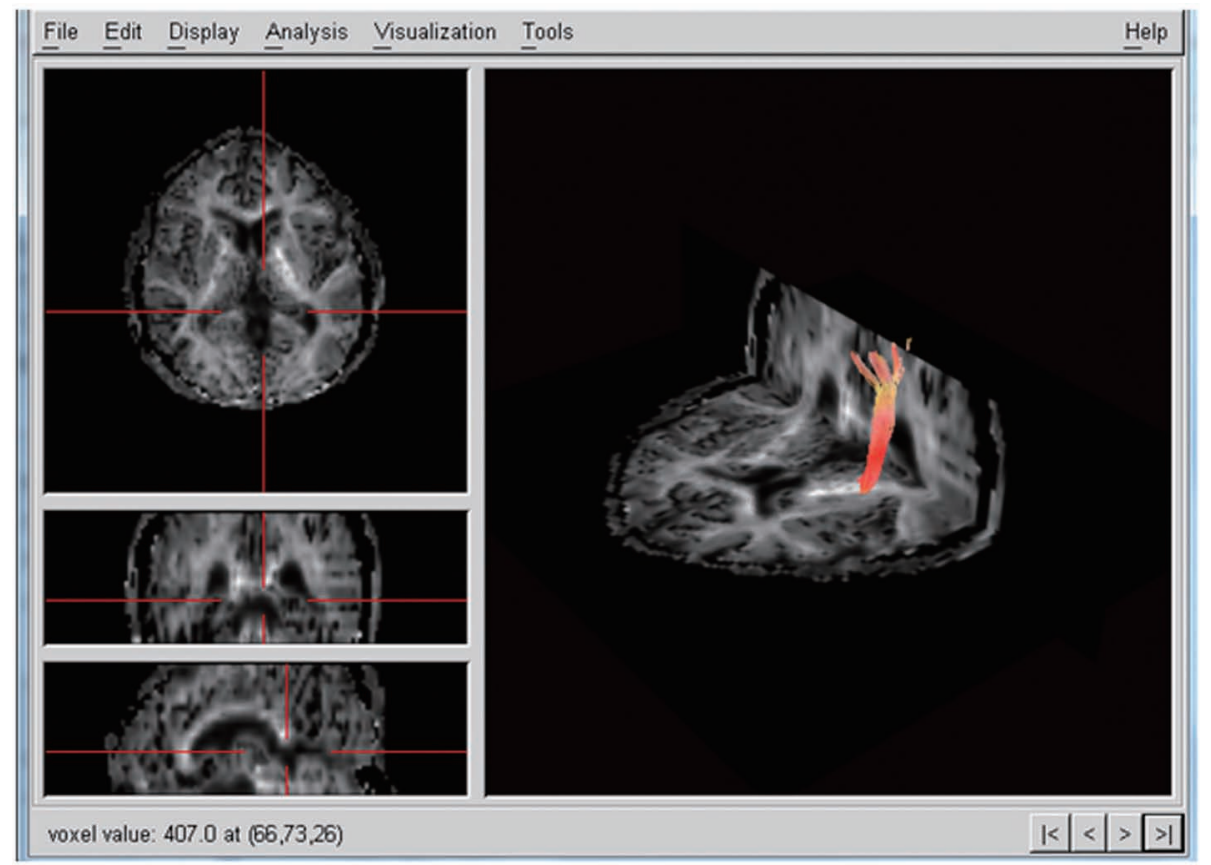

Fig. 4. White matter tractography of the pyramidal tract on a reformatted mean diffusional kurtosis (DK) map created using Volume-One (http://www. volume-one.org/) and dTV software (http://www.ut-radiology.umin.jp/people /masutani/dTV.htm) software.

with stroke, but the results suggest that MDP maps acquired from QSI data provide additional and unique information compared to those obtained from conventional diffusion-weighted sequences (Fig. 5). MR imaging methodologies, and QSI in particular, can also be used to observe neuronal remodeling after brain injury or stroke. ${ }^{43}$ Conventional DTI measurements using the Gaussian diffusion tensor model can only be used to evaluate latestage remodeling that is generally characterized by well-organized axons, whereas QSI can better detect axonal remodeling at the early stage, when ax- 

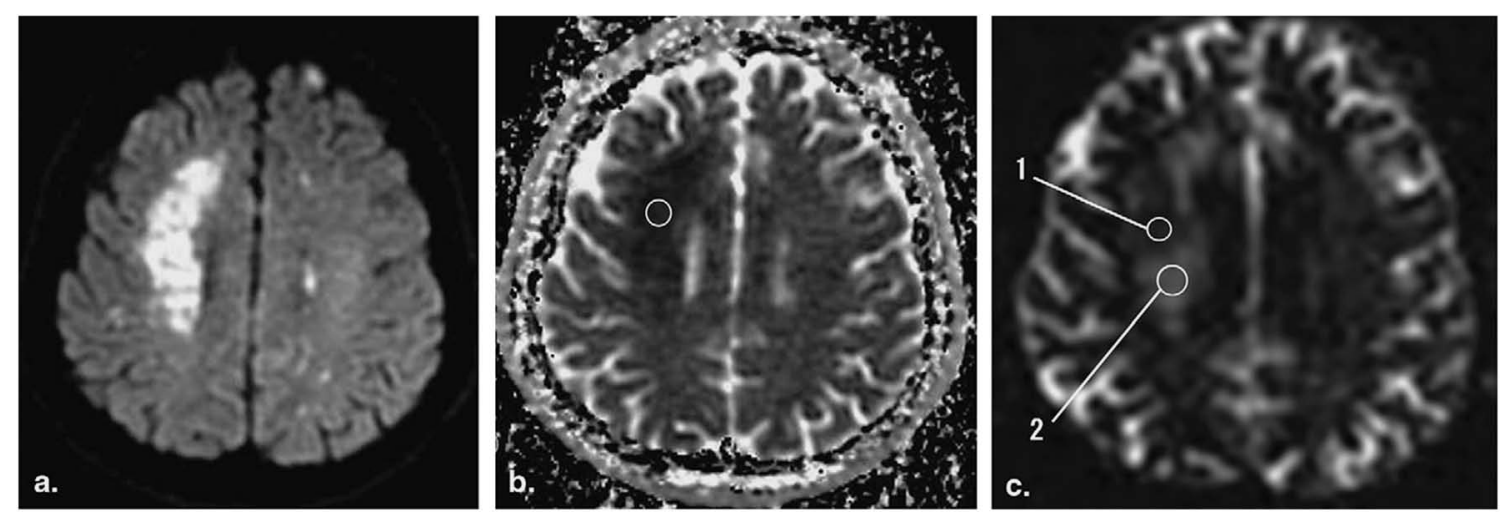

Fig. 5. A case of multiple cerebral infarctions, 120 hours after onset. (a) An axial conventional diffusion-weighted image shows a large hyperintense lesion of the right corona radiata. (b) The corresponding apparent diffusion coefficient (ADC) map shows homogeneous low intensity and decreased ADC value $\left(0.40 \times 10^{-3} \mathrm{~mm}^{2} / \mathrm{s}\right)$ in the lesion (circle= region of interest). (c) The mean displacement (MDP) map shows heterogeneous MDP values in the lesion $(6.2 \mu \mathrm{m}$ in region of interest 1 and $7.9 \mu \mathrm{m}$ in region of interest 2), possibly suggesting a heterogeneous pathologic condition. (Reprinted from Hori M, Motosugi U, Fatima Z, et al. A comparison of mean displacement values using high b-value q-space diffusion-weighted MRI with conventional apparent diffusion coefficients in patients with stroke. Acad Radiol 2011; 18:837-841; with permission.)

ons are less organized and are randomly oriented.

Jensen's group reported substantial increases in MK within ischemic lesions for 3 patients with stroke. ${ }^{29}$ For the first time, they determined the changes in $K_{\|}$and $K_{\perp}$ for human focal cerebral infarction. For lesions with strongly oriented axon bundles, i.e., large contralateral FA values, the change was much greater in $K_{\|}$than $K_{\perp}$, suggesting a large decrease in the intra-axonal diffusivity. Their study illustrates how the application of DKI to the investigation of ischemic stroke can lead to better characterization of the associated, but still not fully understood, changes in water diffusion. DKI thus seems to be a promising method for providing information that conventional diffusion metrics have not been able to show. Hori and associates reported similar results with DKI in a woman with cerebral infarction and gathered additional information, including a more detailed evaluation of pathologic tissue changes. ${ }^{33}$ In this patient, DKI of the stroke lesions showed high intensity in the periphery and a dark dot in the center, whereas conventional DWI produced a homogeneously high signal (Fig. 6). Follow-up MR examination 6 months later showed a hyperintense rim in the area corresponding to the hyperintensity in the DKI. DKI may thus be able to detect the area of gliosis in an ischemic lesion at an early stage after stroke onset. ${ }^{33}$

\section{Multiple sclerosis}

Macroscopic damage is well demonstrated by conventional MR imaging, such as $\mathrm{T}_{2}$-weighted imaging and fluid-attenuated inversion recovery (FLAIR) imaging, but not in occult microscopic pathology. ${ }^{4}$ Several diffusion MR imaging studies have revealed abnormalities of diffusion metrics including FA and ADC in NAWM and normal-appearing gray matter (NAGM) in patients with several types of multiple sclerosis. ${ }^{44-49}$ In these studies, ADC and FA measurements could detect abnormalities with higher sensitivity than conventional MR imaging, such as $T_{2}$-weighted imaging and FLAIR.

Non-Gaussian diffusional metrics have shown promising results in studies with small numbers of patients with multiple sclerosis. High b-value QSI showed higher sensitivity than DTI to pathological changes in NAWM and lesions. ${ }^{19}$ DKI also showed promising results in patients with multiple sclerosis; in these patients, DKI has a clinical advantage because mean kurtosis values are relatively larger in regions of crossing fibers, whereas FA values are lower than surrounding normal white matter (Fig. 7). Therefore, DKI may be a preferable method for evaluating NAWM. Moreover, a recent study showed abnormal FA values in NAGM, ${ }^{50}$ but evaluation of many subjects may be necessary to see significant differences between patients and normal controls. In contrast, the use of DKI to evaluate structural changes in gray matter has been reported in relatively small numbers of subjects. ${ }^{51,52}$ Therefore, DKI may be useful in the evaluation of NAGM in patients with multiple sclerosis. 


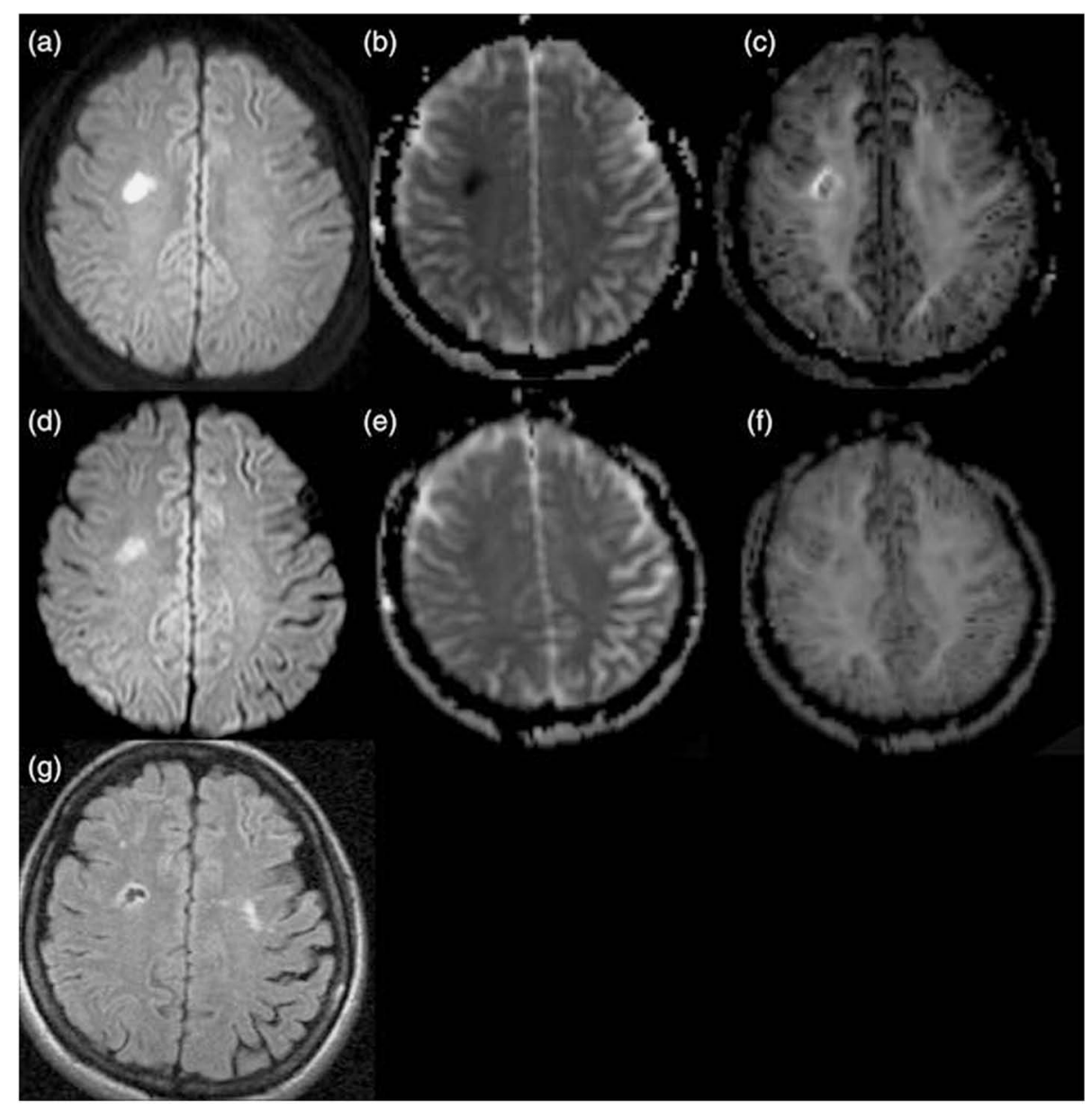

Fig. 6. Magnetic resonance (MR) imaging studies of a patient with stroke. Brain MR imaging demonstrated multiple cerebral infarctions, including a subacute lesion in the right frontal deep white matter, which appeared as a high signal on diffusion-weighted imaging (DWI) (a) and a low signal on the calculated apparent diffusion coefficient (ADC) map (b). However, the corresponding mean diffusional kurtosis (DK) image shows peripherally high intensity and a dark dot within the stroke lesion (c). The mean DK value for the region of high intensity was 1.596 and of the dark dot, 0.591 . Two weeks later, additional MR scanning revealed a homogeneously high signal on both DWI (d) and the ADC map (e) and a partially normalized mean DK (f) in the region corresponding to the ischemic lesion. The mean DK value for the normalized area was 0.842 . In a fluid-attenuated inversion recovery (FLAIR) image taken 6 months later (g), the stroke lesion revealed a low signal in the central cystic portion, with peripheral high intensity, as in the first DK image. (Reprinted from Hori M, Aoki S, Fukunaga I, Suzuki Y, and Masutani Y. A new diffusion metric, diffusion kurtosis imaging, used in the serial examination of a patient with stroke. Acta Radiologica Short Reports 2012;1:1-3. This is an open-access article distributed under the terms of the Creative Commons Attribution License [http://crea tivecommons.org/licenses/by-nc/2.0/]).

\section{Neoplasms of the brain and spine}

An association of the ADC calculated from DWI with tumor cellularity is reported ${ }^{53-55}$ and could aid the differential diagnosis of brain tumors. Although some papers have focused on differentiating high-grade gliomas and lymphoma, whose cellularities are different, ${ }^{53,56-60}$ ADC values overlap be- tween gliomas and lymphoma. ${ }^{61}$

Murakami's team reported that use of the ADC can improve accuracy in grading astrocytic tumors, using a combination of minimum ADCs and ADC difference values (2-parameter method). ${ }^{62}$ However, the clinical usefulness of this technique remains limited because of overlap in the regional 


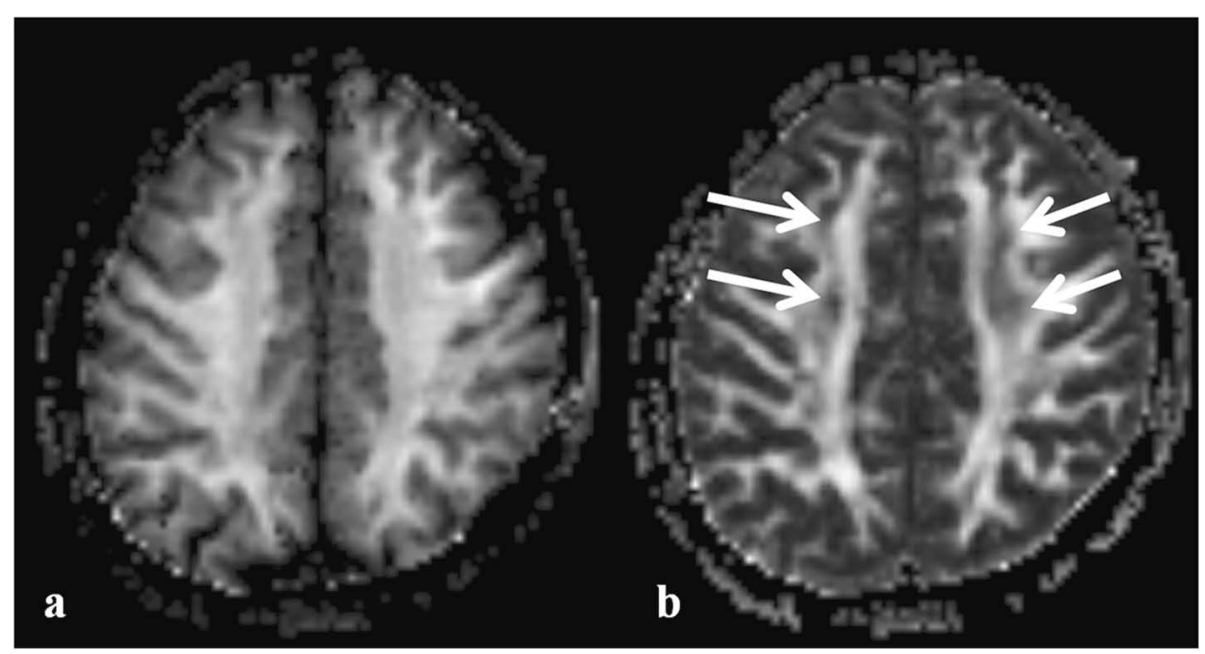

Fig. 7. A mean diffusional kurtosis (DK) image (a) showed no signal loss at white matter fiber crossing points, unlike the corresponding fractional anisotropy (FA) image (b, arrows). DK imaging may therefore be a better tool for evaluating white matter lesions than FA.

ADC values among gliomas of differing grades, ${ }^{63,64}$ in particular between tumors of WHO grades II and III. The ADC was thought to be inversely correlated with tumor cellularity because higher grade tumors have been found to have higher cellularity than tumors of lower grade. Though there are few reports of the use of non-Gaussian diffusional techniques to evaluate brain tumors, the techniques seem promising by showing better results than conventional diffusional metrics. Raab and colleagues showed that DKI data are superior to ADC in differentiating glioma grades. ${ }^{65}$ In their results, mean kurtosis values increased with increasing grade (Fig. 8), whereas ADCs tended to decrease with tumor grade and FA values did not differ significantly among tumor groups. Significant differences between astrocytoma of WHO grades II and III were demonstrated only by DK values. ${ }^{65} \mathrm{In}$ addition, Van Cauter and associates reported significant differences in kurtosis parameters between high- and low-grade gliomas using mean, radial, and axial kurtosis values on a 3T MR imaging scanner. ${ }^{66}$ In this report, only kurtosis MR imaging parameters showed significant differences between different grades of gliomas; FA and mean diffusivity did not differ significantly between them.

With respect to spine and spinal cord tumors, Hori's group reported the MDP values of normal spinal cord (6.57 \pm 0.52$)$, cerebrospinal fluid (CSF) (17.6 \pm 2.75$)$, and tumor (neurinoma) parenchyma $(8.49 \pm 2.09)(\mu \mathrm{m}$, mean \pm standard deviation) obtained using QSI with a maximum q value of 836.9 $\mathrm{cm}^{-1}$ on a 1.5T MR imaging scanner (Fig. 9). In general, MDP maps did not correlate well with cor-

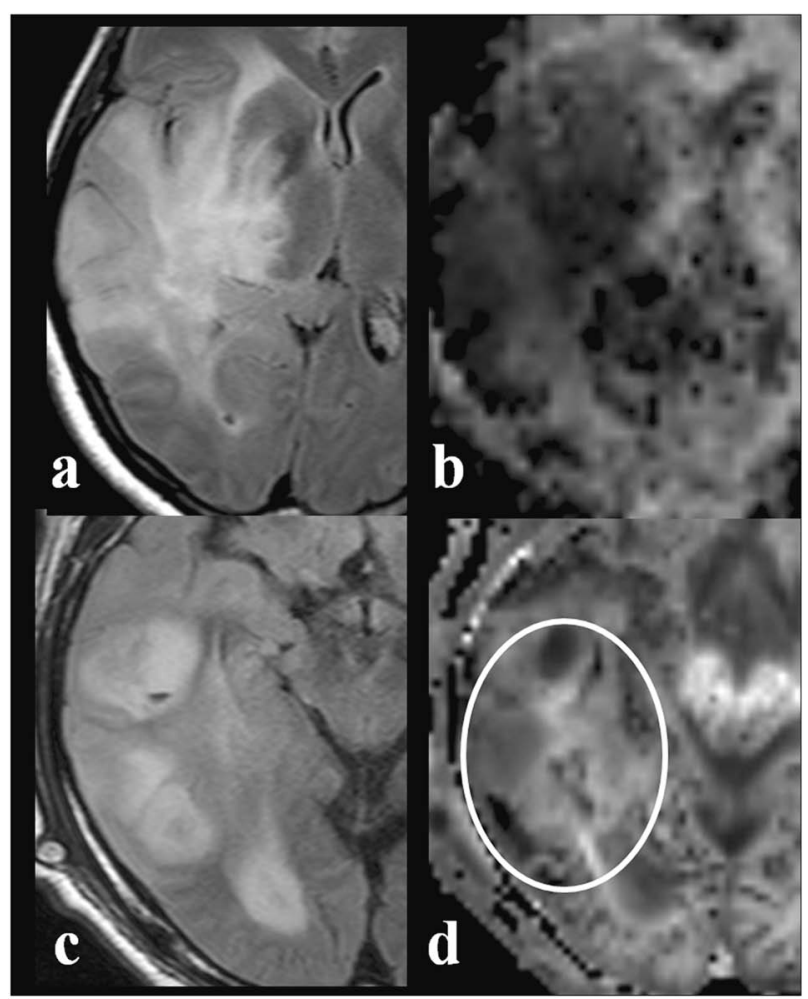

Fig. 8. Patients with low- (a, b) and high-grade glioma (c, d). Both fluid-attenuated inversion recovery (FLAIR) images (a, c) show abnormally high intensities within the tumor. On mean diffusional kurtosis (DK) maps, high intratumoral DK values are prominent in the high-grade glioma (d, circle), but not in the low-grade tumor (b).

responding ADC maps at the pathologic lesions. Therefore, they concluded that this technique has the potential to provide additional clinical informa- 


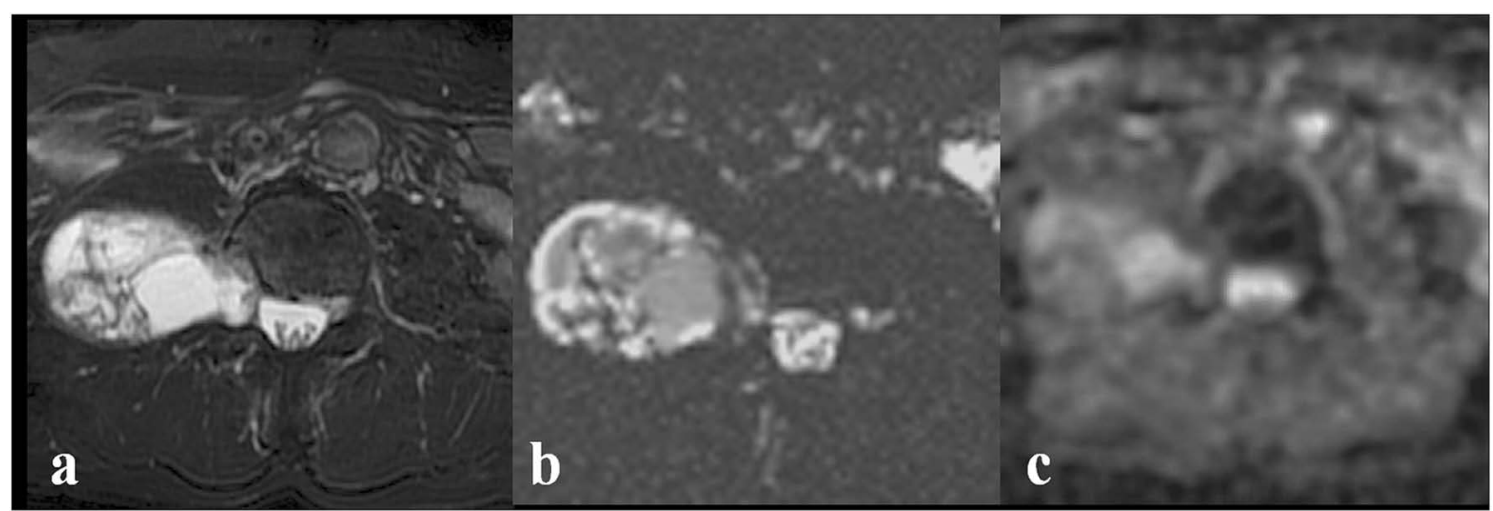

Fig. 9. Neurinoma in a 69-year-old man. An axial $\mathrm{T}_{2}$-weighted image (a) shows slightly high signal intensity in the cervical spinal cord. The mean displacement (MDP) map (b) shows internal structures of the cervical spinal cord in detail. The degradation of image quality in the corresponding apparent diffusion coefficient (ADC) map (c) derived from conventional diffusion-weighted imaging (DWI) at the same location makes interpretation difficult. (Reprinted from Hori M, Motosug U, Fatima Z, Shigame K, Araki T. Mean displacement map of spine and spinal cord disorders using high b-value q-space imaging-feasibility study. Acta Radiol 2011; 52:1155-1158; Copyright (C) 2011 by Royal Society of Medicine Press, UK.)

tion to that obtained with conventional MR imaging. ${ }^{31}$

\section{Neuropsychiatric disease}

In the past few decades, advanced MR imaging techniques, such as functional MR imaging, MR spectroscopy, and diffusion-tensor imaging, have shown correlations between functional, chemical, and structural changes and psychiatric disorders. ${ }^{67}$ The conventional DTI technique is reported to identify white matter disruptions in specific regions, such as the cingulum, uncinate fasciculus, arcuate fasciculus, and corpus callosum, ${ }^{68,69}$ and decreased FA has been observed in lesions in patients with schizophrenia. ${ }^{68,69}$ Moreover, decreased FA has been observed in the frontal tract and corpus callosum in patients with bipolar disorder. ${ }^{70,71}$ In general, abnormal white matter connections are common in patients with schizophrenia and affective disorders ${ }^{67}$ There are small numbers of studies in this field using non-Gaussian DWI. Using DKI measurements, Helpern's group reported less development of the frontal white matter in patients with attention-deficit hyperactivity disorder than in typically developing controls. ${ }^{51}$ In addition, gray matter microstructure also lacked the significant age-related increase in complexity seen in controls; only kurtosis measures could detect this difference. Therefore, the authors concluded that DKI provides information about changes in tissue microstructure that is more sensitive than and complementary to that provided by conventional diffusion imaging methods. In patients with Alzheimer disease and mild cognitive impairment, histogram analysis of mean diffusivity, FA, and MK suggested a continuous spectrum of changes in tissue microstructure during normal aging and during the progression to Alzheimer disease, though the study population was small. ${ }^{72}$ A clinical QSI study also showed the usefulness of QSI parameters for assessing Alzheimer disease. Using tract-based analysis, Taoka and colleagues identified decreased maximal probabilities of PDF, broader FWHM, and sharper MK in the bilateral uncinate fascicles and posterior cingulum in patients with Alzheimer disease compared with normal volunteers. They speculated that the results may reflect altered permeability or damage in the cell membrane, myelin, or both. ${ }^{73}$

\section{Changes in the brain and spinal cord with aging and degenerative disease}

DTI has been also used to study degenerative changes in healthy and diseased brain. The most common findings from DTI research related to aging are age-related increases in mean diffusivity and decreases in FA. ${ }^{74}$ As for non-Gaussian DWI, various diffusivity parameters estimated by the DKI model were powerful tools for assessing changes in tissue microstructure and detecting developmental changes in transgenic rat pups in a Huntington disease model. ${ }^{75}$ Moreover, Zhuo and associates showed that DKI can detect microstructural changes associated with reactive astrogliosis following traumatic brain injury that may be missed when using standard DTI parameters. ${ }^{76} \mathrm{FA}$ of the cingulate 

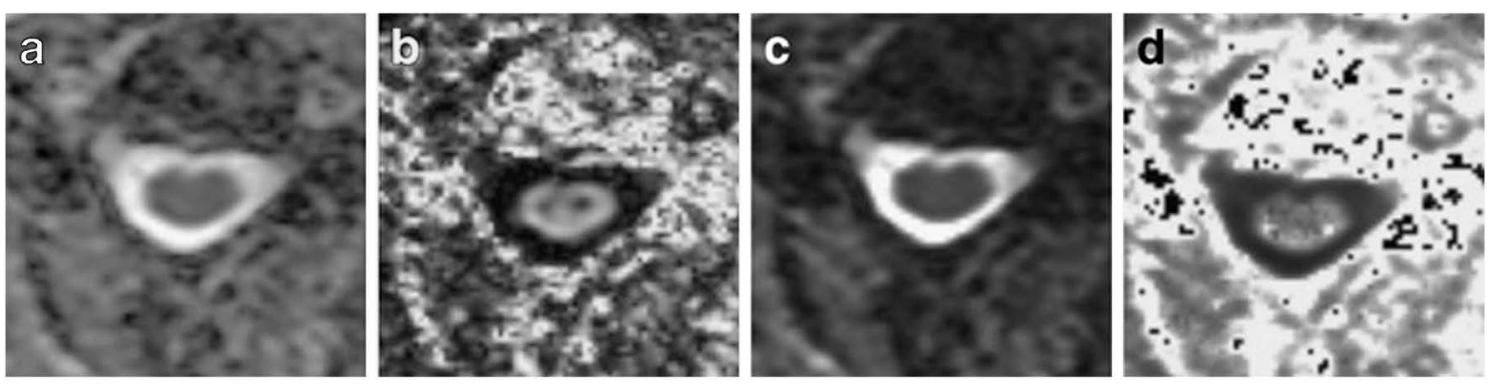

Fig. 10. Diffusion metric maps of the cervical spinal cord in the axial plane. Apparent diffusion coefficient (a), fractional anisotropy (b), full width at half maximum of the probability density function (c), and mean diffusional kurtosis (d) at the C3-C4 vertebral level. (Reprinted from Hori M, Fukunaga I, Masutani Y, et al. New diffusion metrics for spondylotic myelopathy at an early clinical stage. Eur Radiol 2012; 22:1797-1802. This is an open-access article distributed under the terms of the Creative Commons Attribution License [http://creativecommons.org/licenses/by-nc/2.0/]).

fiber tracts is reduced in patients with Parkinson disease with and without dementia compared with normal controls, ${ }^{77}$ and Wang's team showed that the mean kurtosis of the ipsilateral substantia nigra performed best in diagnosing Parkinson disease (sensitivity, 0.92; specificity, 0.87), compared with conventional diffusion metrics including FA, mean diffusivity, axial diffusivity, and radial diffusivity. ${ }^{78}$ Therefore, non-Gaussian DWI and DKI, in particular, may be promising for the evaluation of degenerative changes in vivo.

Studies assessing the spinal cord have shown similar results to those seen in the brain, including the general observation that FA decreases with the progression of degenerative changes. ${ }^{79,80}$ Using non-Gaussian DWI, Hori's group reported that mean DK and MPD values in the spinal cord may be highly sensitive indicators of microstructural changes and damage in early cervical spondylosis (Fig. 10). ${ }^{34}$ In general, non-Gaussian metrics may provide additional information for the assessment of degenerative changes and disease in the brain and spinal cord.

\section{Current Limitations and Future Challenges}

The most important hurdle to using QSI as a daily clinical tool is the long scan time. Acquisition of a QSI dataset that covers the whole brain with $3-\mathrm{mm}$ isotropic spatial resolution requires $10 \mathrm{~min}$. Though scan time is shorter for DKI than QSI, larger numbers of MPGs will lead to better contrast in the DK map. Farrell's group suggested that acquisition of images at fewer b-values and using a fitting and interpolation procedure could reduce total scan time for a QSI dataset. ${ }^{21}$ Otherwise, some advanced technique, such as compressed sensing, ${ }^{81}$ will be needed to overcome the problem.
A second problem, the appropriate registration method to use with DWI data obtained with high bvalues (b $>3000 \mathrm{~s} / \mathrm{mm}^{2}$ ), remains under investigation. Existing registration methods for DTI are not effective at these values and may occasionally lead to misregistration. Therefore, a specific method should be developed for high b-value datasets.

\section{Conclusions}

Although the methodologies of non-Gaussian diffusion analysis, including QSI, provide a better theoretical basis on which to address the complicated diffusion of water in white matter, experimentally, they are time consuming and require strong computational power. ${ }^{41}$ They cannot replace the fast acquisition and analysis of DTI, but they most certainly can yield complementary information not obtained with DTI. ${ }^{41}$ DKI is an expansion of DTI and requires less hardware and software than QSI and, so, will be a better tool than QSI for clinical use.

\section{References}

1. Sotak $\mathrm{CH}$. The role of diffusion tensor imaging in the evaluation of ischemic brain injury-a review. NMR Biomed 2002; 15:561-569.

2. Basser PJ, Mattiello J, LeBihan D. MR diffusion tensor spectroscopy and imaging. Biophys J 1994; 66:259-267.

3. Fung SH, Roccatagliata L, Gonzalez RG, Schaefer PW. MR diffusion imaging in ischemic stroke. Neuroimaging Clin N Am 2011; 21:345-377.

4. Inglese M, Bester M. Diffusion imaging in multiple sclerosis: research and clinical implications. NMR Biomed 2010; 23:865-872.

5. Morita N, Harada M, Otsuka H, Melhem ER, Nishitani H. Clinical application of MR spectros- 
copy and imaging of brain tumor. Magn Reson Med Sci 2010; 9:167-175.

6. Zou QG, Xu HB, Liu F, Guo W, Kong XC, Wu Y. In the assessment of supratentorial glioma grade: the combined role of multivoxel proton MR spectroscopy and diffusion tensor imaging. Clin Radiol 2011; 66:953-960.

7. Deng Z, Yan Y, Zhong D, et al. Quantitative analysis of glioma cell invasion by diffusion tensor imaging. J Clin Neurosci 2010; 17:1530-1536.

8. Morikawa M, Kiuchi $\mathrm{K}$, Taoka $\mathrm{T}$, Nagauchi $\mathrm{K}$, Kichikawa K, Kishimoto T. Uncinate fasciculuscorrelated cognition in Alzheimer's disease: a diffusion tensor imaging study by tractography. Psychogeriatrics 2010; 10:15-20.

9. Taoka T, Morikawa M, Akashi T, et al. Fractional anisotropy-threshold dependence in tract-based diffusion tensor analysis: evaluation of the uncinate fasciculus in Alzheimer disease. AJNR Am J Neuroradiol 2009; 30:1700-1703.

10. Sato K, Aoki S, Iwata NK, et al. Diffusion tensor tract-specific analysis of the uncinate fasciculus in patients with amyotrophic lateral sclerosis. Neuroradiology 2010; 52:729-733.

11. Kumar A, Sundaram SK, Sivaswamy L, et al. Alterations in frontal lobe tracts and corpus callosum in young children with autism spectrum disorder. Cereb Cortex 2010; 20:2103-2113.

12. Sundaram SK, Kumar A, Makki MI, Behen ME, Chugani HT, Chugani DC. Diffusion tensor imaging of frontal lobe in autism spectrum disorder. Cereb Cortex 2008; 18:2659-2665.

13. Nenadic I, Wagner G, Güllmar D, et al. ADC changes in schizophrenia: a diffusion-weighted imaging study. Eur Arch Psychiatry Clin Neurosci 2011; 261:213-216.

14. Rametti G, Junqué C, Falcón C, et al. A voxelbased diffusion tensor imaging study of temporal white matter in patients with schizophrenia. Psychiatry Res 2009; 171:166-176.

15. Lätt J, Nilsson M, van Westen D, Wirestam R, Ståhlberg F, Brockstedt S. Diffusion-weighted MRI measurements on stroke patients reveal waterexchange mechanisms in sub-acute ischaemic lesions. NMR Biomed 2009; 22:619-628.

16. Assaf Y, Cohen Y. Structural information in neuronal tissue as revealed by q-space diffusion NMR spectroscopy of metabolites in bovine optic nerve. NMR Biomed 1999; 12:335-344.

17. Assaf Y, Cohen Y. Assignment of the water slowdiffusing component in the central nervous system using q-space diffusion MRS: implications for fiber tract imaging. Magn Reson Med 2000; 43:191-199.

18. Assaf Y, Mayk A, Cohen Y. Displacement imaging of spinal cord using q-space diffusion-weighted MRI. Magn Reson Med 2000; 44:713-722.

19. Assaf Y, Ben-Bashat D, Chapman J, et al. High bvalue q-space analyzed diffusion-weighted MRI: application to multiple sclerosis. Magn Reson Med
2002; 47:115-126.

20. Cohen Y, Assaf Y. High b-value q-space analyzed diffusion-weighted MRS and MRI in neuronal tissues-a technical review. NMR Biomed 2002; 15:516-542.

21. Farrell JA, Smith SA, Gordon-Lipkin EM, Reich DS, Calabresi PA, van Zijl PC. High b-value qspace diffusion-weighted MRI of the human cervical spinal cord in vivo: feasibility and application to multiple sclerosis. Magn Reson Med 2008; 59: 1079-1089.

22. Fatima Z, Motosugi U, Hori M, et al. q-space imaging (QSI) of the brain: comparison of displacement parameters by QSI and DWI. Magn Reson Med Sci 2010; 9:109-110.

23. Hikishima K, Yagi K, Numano T, et al. Volumetric q-space imaging by $3 \mathrm{D}$ diffusion-weighted MRI. Magn Reson Imaging 2008; 26:437-445.

24. Assaf Y, Chapman J, Ben-Bashat D, et al. White matter changes in multiple sclerosis: correlation of q-space diffusion MRI and 1H MRS. Magn Reson Imaging 2005; 23:703-710.

25. Lätt $\mathrm{J}$, Nilsson $\mathrm{M}$, Wirestam $\mathrm{R}$, et al. In vivo visualization of displacement-distribution-derived parameters in q-space imaging. Magn Reson Imaging 2008; 26:77-87.

26. Hori M, Motosugi U, Fatima Z, et al. A comparison of mean displacement values using high bvalue q-space diffusion-weighted MRI with conventional apparent diffusion coefficients in patients with stroke. Acad Radiol 2011; 18:837-841.

27. Jensen JH, Helpern JA. MRI quantification of non-Gaussian water diffusion by kurtosis analysis. NMR Biomed 2010; 23:698-710.

28. Jensen JH, Helpern JA, Ramani A, Lu H, Kaczynski K. Diffusional kurtosis imaging: the quantification of non-Gaussian water diffusion by means of magnetic resonance imaging. Magn Reson Med 2005; 53:1432-1440.

29. Jensen JH, Falangola MF, Hu C, et al. Preliminary observations of increased diffusional kurtosis in human brain following recent cerebral infarction. NMR Biomed 2011; 24:452-457.

30. Fatima Z, Motosugi U, Hori M, et al. High b-value q-space analyzed diffusion-weighted MRI using 1.5 tesla clinical scanner; determination of displacement parameters in the brains of normal versus multiple sclerosis and low-grade glioma subjects. J Neuroimaging. 2012; 22:279-284.

31. Hori M, Motosugi U, Fatima Z, Ishigame K, Araki T. Mean displacement map of spine and spinal cord disorders using high b-value q-space imagingfeasibility study. Acta Radiol 2011; 52:1155-1158.

32. Tabesh A, Jensen JH, Ardekani BA, Helpern JA. Estimation of tensors and tensor-derived measures in diffusional kurtosis imaging. Magn Reson Med 2011; 65:823-836.

33. Hori M, Aoki S, Fukunaga I, et al. A new diffusion metric, diffusion kurtosis imaging, used in the seri- 
al examination of a patient with stroke. Acta Radiologica Short Reports 2012; 1:1-3.

34. Hori M, Fukunaga I, Masutani Y, et al. New diffusion metrics for spondylotic myelopathy at an early clinical stage. Eur Radiol 2012; 22:1797-1802.

35. Marks MP, Tong DC, Beaulieu C, Albers GW, de Crespigny A, Moseley ME. Evaluation of early reperfusion and i.v. tPA therapy using diffusionand perfusion-weighted MRI. Neurology 1999; 52: 1792-1798.

36. Schlaug G, Siewert B, Benfield A, Edelman RR, Warach S. Time course of the apparent diffusion coefficient (ADC) abnormality in human stroke. Neurology 1997; 49:113-119.

37. Fiehler J, Fiebach JB, Gass A, et al. Diffusionweighted imaging in acute stroke-a tool of uncertain value? Cerebrovasc Dis 2002; 14:187-196.

38. Aoki S, Masutani Y, Abe O. [Magnetic resonance diffusion tractography in the brain-its application and limitation]. Brain Nerve 2007; 59:467-476. [Article in Japanese]

39. Kunimatsu A, Aoki S, Masutani Y, et al. The optimal trackability threshold of fractional anisotropy for diffusion tensor tractography of the corticospinal tract. Magn Reson Med Sci 2004; 3:11-17.

40. Morita N, Harada M, Uno M, Furutani K, Nishitani H. Change of diffusion anisotropy in patients with acute cerebral infarction using statistical parametric analysis. Radiat Med 2006; 24: 253-259.

41. Assaf Y, Pasternak O. Diffusion tensor imaging (DTI)-based white matter mapping in brain research: a review. J Mol Neurosci 2008; 34:51-61.

42. Assaf Y, Mayk A, Eliash S, Speiser Z, Cohen Y. Hypertension and neuronal degeneration in excised rat spinal cord studied by high-b value q-space diffusion magnetic resonance imaging. Exp Neurol 2003; 184:726-736.

43. Jiang Q, Zhang ZG, Chopp M. MRI evaluation of white matter recovery after brain injury. Stroke 2010; 41(10 Suppl):S112-S113.

44. Rocca MA, Cercignani M, Iannucci G, Comi G, Filippi M. Weekly diffusion-weighted imaging of normal-appearing white matter in MS. Neurology 2000; 55:882-884.

45. Guo AC, MacFall JR, Provenzale JM. Multiple sclerosis: diffusion tensor MR imaging for evaluation of normal-appearing white matter. Radiology 2002; 222:729-736.

46. Rovaris M, Bozzali M, Iannucci G, et al. Assessment of normal-appearing white and gray matter in patients with primary progressive multiple sclerosis: a diffusion-tensor magnetic resonance imaging study. Arch Neurol 2002; 59:1406-1412.

47. Rovaris M, Gallo A, Valsasina P, et al. Short-term accrual of gray matter pathology in patients with progressive multiple sclerosis: an in vivo study using diffusion tensor MRI. Neuroimage 2005; 24: 1139-1146.
48. Rocca MA, Iannucci G, Rovaris M, Comi G, Filippi M. Occult tissue damage in patients with primary progressive multiple sclerosis is independent of $\mathrm{T}_{2}$-visible lesions-a diffusion tensor $\mathrm{MR}$ study. J Neurol 2003; 250:456-460.

49. Ciccarelli O, Werring DJ, Wheeler-Kingshott CA, et al. Investigation of MS normal-appearing brain using diffusion tensor MRI with clinical correlations. Neurology 2001; 56:926-933.

50. Calabrese M, Rinaldi F, Seppi D, et al. Cortical diffusion-tensor imaging abnormalities in multiple sclerosis: a 3-year longitudinal study. Radiology 2011; 261:891-898.

51. Helpern JA, Adisetiyo V, Falangola MF, et al. Preliminary evidence of altered gray and white matter microstructural development in the frontal lobe of adolescents with attention-deficit hyperactivity disorder: a diffusional kurtosis imaging study. J Magn Reson Imaging 2011; 33:17-23.

52. Falangola MF, Jensen JH, Babb JS, et al. Agerelated non-Gaussian diffusion patterns in the prefrontal brain. J Magn Reson Imaging 2008; 28: 1345-1350.

53. Guo AC, Cummings TJ, Dash RC, Provenzale JM. Lymphomas and high-grade astrocytomas: comparison of water diffusibility and histologic characteristics. Radiology 2002; 224:177-183.

54. Sugahara T, Korogi Y, Kochi M, et al. Usefulness of diffusion-weighted MRI with echo-planar technique in the evaluation of cellularity in gliomas. $\mathrm{J}$ Magn Reson Imaging 1999; 9:53-60.

55. Gupta RK, Cloughesy TF, Sinha U, et al. Relationships between choline magnetic resonance spectroscopy, apparent diffusion coefficient and quantitative histopathology in human glioma. J Neurooncol 2000; 50:215-226.

56. Kitis O, Altay H, Calli C, Yunten N, Akalin T, Yurtseven T. Minimum apparent diffusion coefficients in the evaluation of brain tumors. Eur $\mathbf{J}$ Radiol 2005; 55:393-400.

57. Yamasaki F, Kurisu K, Satoh K, et al. Apparent diffusion coefficient of human brain tumors at MR imaging. Radiology 2005; 235:985-991.

58. Horger M, Fenchel M, Nägele T, et al. Water diffusivity: comparison of primary CNS lymphoma and astrocytic tumor infiltrating the corpus callosum. AJR Am J Roentgenol 2009; 193:1384-1387.

59. Toh CH, Castillo M, Wong AM, et al. Primary cerebral lymphoma and glioblastoma multiforme: differences in diffusion characteristics evaluated with diffusion tensor imaging. AJNR Am J Neuroradiol 2008; 29:471-475.

60. Doskaliyev A, Yamasaki F, Ohtaki M, et al. Lymphomas and glioblastomas: differences in the apparent diffusion coefficient evaluated with high bvalue diffusion-weighted magnetic resonance imaging at 3T. Eur J Radiol 2012; 81:339-344.

61. Server A, Kulle B, Maehlen J, et al. Quantitative apparent diffusion coefficients in the characteriza- 
tion of brain tumors and associated peritumoral edema. Acta Radiol 2009; 50:682-689.

62. Murakami R, Hirai T, Sugahara T, et al. Grading astrocytic tumors by using apparent diffusion coefficient parameters: superiority of a one- versus two-parameter pilot method. Radiology 2009; 251: 838-845.

63. Kono $\mathrm{K}$, Inoue $\mathrm{Y}$, Nakayama $\mathrm{K}$, et al. The role of diffusion-weighted imaging in patients with brain tumors. AJNR Am J Neuroradiol 2001; 22:10811088.

64. Zimmerman RD. Is there a role for diffusionweighted imaging in patients with brain tumors or is the "bloom off the rose"? AJNR Am J Neuroradiol 2001; 22:1013-1014.

65. Raab P, Hattingen E, Franz K, Zanella FE, Lanfermann H. Cerebral gliomas: diffusional kurtosis imaging analysis of microstructural differences. Radiology 2010; 254:876-881.

66. Van Cauter S, Veraart J, Sijbers J, et al. Gliomas: diffusion kurtosis MR imaging in grading. Radiology 2012; 263:492-501.

67. Agarwal N, Port JD, Bazzocchi M, Renshaw PF. Update on the use of MR for assessment and diagnosis of psychiatric diseases. Radiology 2010; 255: 23-41.

68. Kanaan RA, Kim JS, Kaufmann WE, Pearlson GD, Barker GJ, McGuire PK. Diffusion tensor imaging in schizophrenia. Biol Psychiatry 2005; 58:921-929.

69. Kunimatsu N, Aoki S, Kunimatsu A, et al. Tractspecific analysis of white matter integrity disruption in schizophrenia. Psychiatry Res 2012; 201: 136-143.

70. Adler CM, Holland SK, Schmithorst V, et al. Abnormal frontal white matter tracts in bipolar disorder: a diffusion tensor imaging study. Bipolar Disord 2004; 6:197-203.

71. Haznedar MM, Roversi F, Pallanti S, et al. Fronto-thalamo-striatal gray and white matter volumes and anisotropy of their connections in bipolar spectrum illnesses. Biol Psychiatry 2005; 57: 733-742.
72. Lu H, Jensen $\mathrm{JH} \mathrm{J,} \mathrm{Hu} \mathrm{C,} \mathrm{et} \mathrm{al.} \mathrm{Alterations} \mathrm{in}$ cerebral microstructual integrity in normal aging and in Alzheimer's disease: a multi-contrast diffusion MRI study. Proceedings of the 14th Annual Meeting of ISMRM. Seattle, Washington, USA, 2006; 723 .

73. Taoka T, Sakamoto M, Akashi T, et al. q-space imaging in the clinical cases with Alzheimer disease: analysis of fibers in the limbic system. Proceedings of the 20th Annual Meeting of ISMRM. Melbourne, Australia, 2012; 983.

74. Inano S, Takao H, Hayashi N, Abe O, Ohtomo K. Effects of age and gender on white matter integrity. AJNR Am J Neuroradiol 2011; 32:2103-2109.

75. Blockx I, De Groof G, Verhoye M, et al. Microstructural changes observed with DKI in a transgenic Huntington rat model: evidence for abnormal neurodevelopment. Neuroimage 2012; 59:957-967.

76. Zhuo J, Xu S, Proctor JL, et al. Diffusion kurtosis as an in vivo imaging marker for reactive astrogliosis in traumatic brain injury. Neuroimage 2012; 59: 467-477.

77. Kamagata K, Motoi Y, Abe O, et al. White matter alteration of the cingulum in Parkinson disease with and without dementia: evaluation by diffusion tensor tract-specific analysis. AJNR Am J Neuroradiol 2012; 33:890-895.

78. Wang JJ, Lin WY, Lu CS, et al. Parkinson disease: diagnostic utility of diffusion kurtosis imaging. Radiology 2011; 261:210-217.

79. Mamata H, Jolesz FA, Maier SE. Apparent diffusion coefficient and fractional anisotropy in spinal cord: age and cervical spondylosis-related changes. J Magn Reson Imaging 2005; 22:38-43.

80. Hori M, Okubo T, Aoki S, Kumagai H, Araki T. Line scan diffusion tensor MRI at low magnetic field strength: feasibility study of cervical spondylotic myelopathy in an early clinical stage. J Magn Reson Imaging 2006; 23:183-188.

81. Menzel MI, Tan ET, Khare K, et al. Accelerated diffusion spectrum imaging in the human brain using compressed sensing. Magn Reson Med 2011; $66: 1226-1233$. 